\title{
Development and Characterization of a ssDNA-based Aptamer that Selectively Targets Epithelial Carcinoma Cells
}

\section{Nianxi Zhao ${ }^{1}$, Yimin $\mathrm{Ge}^{1}$, Paul J Chiao ${ }^{2}$ and Youli $\mathrm{Zu}^{1^{*}}$}

${ }^{1}$ Department of Pathology and Genomic Medicine, Houston Methodist Hospital, and Cancer Pathology Laboratory, Houston Methodist Research Institute, 6565 Fannin Street, Houston, TX 77030, USA

${ }^{2}$ Department of Molecular and Cellular Oncology, The University of Texas MD Anderson Cancer Center, 1515 Holcombe Blvd, Unit 108, Houston, TX 77030, USA

"Corresponding author: Youli Zu, Department of Pathology and Genomic Medicine Houston Methodist Hospital 6565 Fannin Street Houston, TX 77030, USA, Tel: 7134414460; Fax: 7134411565; E-mail: yzu@houstonmethodist.org

Rec date: Oct 27, 2014, Acc date: Nov 18, 2014, Pub date: Nov 25, 2014

Copyright: @ 2014 Zhao N, et al. This is an open-access article distributed under the terms of the Creative Commons Attribution License, which permits unrestricted use, distribution, and reproduction in any medium, provided the original author and source are credited.

\begin{abstract}
Epithelial cellular adhesion molecule (EpCAM) is a biomarker highly expressed on the surface of epithelial carcinoma cells. Consequently, it has been widely used for detection of carcinoma tumors and isolation of individual circulating cancer cells and EpCAM-targeting therapeutics are undergoing pre-clinical and clinical testing against several types of carcinomas. Aptamers are a new class of small oligonucleotide ligands that bind to their targets with high affinity and specificity, and are thus termed "chemical antibodies." In comparison to protein antibodies, aptamers are smaller in size, which improves their tissue penetration potential, and elicit few to no immunogenic responses. Furthermore, our previous studies revealed that ssDNA, compared to RNA aptamers are significantly more stable in human serum and thus, are suitable for in vivo use. In this study, by using a hybrid cell-biomarker selection approach, we developed ssDNA-based aptamers specific for EpCAM. We identified a dominant aptamer sequence $(58.7 \%$ of $>0.5$ million sequence reads) that contained both loop and stem structures, and specifically targeted EpCAM-expressing carcinoma cells with high binding affinity $(\mathrm{Kd}=12 \mathrm{nM})$. Validation studies indicated that the newly synthesized aptamer targeted a variety of cultured carcinoma cells, including pancreatic, breast, ovarian, prostate, and colon cancer cells, but did not react with cultured EpCAM-negative leukemia and lymphoma cells. In addition, the aptamer recognized EpCAM-expressing, patient-derived primary normal and carcinoma cells. Finally, the aptamer selectively targeted carcinoma cells in complex cell mixtures, and showed no reactivity to mononucleated or red blood cells, demonstrating its suitability for therapeutic in vivo use.
\end{abstract}

Keywords: EpCAM; Aptamer; Epithelial cancer cells; Carcinoma diagnostics

\section{Introduction}

The epithelial cell adhesion molecule (EpCAM or CD326) is a 40$\mathrm{kDa}$ transmembrane glycoprotein expressed on the surface of most epithelial cells $[1,2]$. It is also strongly expressed in many types of carcinomas, including colon and rectum, prostate, liver and esophagus, lung, head and neck, pancreas, and breast $[3,4]$. Importantly, EpCAM expression in normal epithelia is generally lower than that in carcinoma cells, and most hematopoietic tumors, such as leukemias and lymphomas, are negative for EpCAM expression [5]. Therefore, EpCAM expression is a hallmark of epithelial tumors, and is widely used in carcinoma diagnostics [6-9].

Aptamers are small molecule probes composed of short, singlestranded RNA or ssDNA oligonucleotide sequences that range from 30 to 60 bases in length and specifically recognize their targets on the basis of their unique 3-dimensional (3D) structures [10-15]. Aptamers are synthesized from RNA or ssDNA libraries via a defined experimental process called Systematic Evolution of Ligands by Exponential enrichment (SELEX) [16-19]. To date, a large variety of aptamers had been developed through SELEX technology, including aptamers targeting small molecules (such as dyes, metal ions, amino acids, and short peptides), biologic macromolecules (nucleic acids and proteins), molecular complexes, viruses, bacteria, live cells, and whole organisms [20-25]. Aptamers combine nanomolar range target- binding affinities with exquisite target specificity, which makes them a versatile tool for diagnostics, in vivo imaging, and targeted therapeutics [12,26-29]. Moreover, compared to protein antibodies, aptamers offer unparalleled advantages, as they can be chemically synthesized through a simple process, modified with a variety of functional groups and/or imaging reporters, and elicit little to no immunogenicity or toxicity in preclinical studies [30-34]. Taking advantage of these aptamer properties, our group recently demonstrated that synthetic aptamers can be successfully used as specific probes for flow cytometric analysis in vitro $[35,36]$ and exhibit high tumor penetrating abilities and rapid tumor cells binding in vivo [37].

In the present study, we employed a hybrid cell-biomarker SELEX technology to develop ssDNA-based aptamers that target EpCAM, a carcinoma cell surface marker. The hybrid aptamer selection process included utilization of immortalized cell lines, patient-derived primary cells, and recombinant proteins in order to achieve high target specificity and affinity. The resultant aptamer was then used to detect various types of cultured carcinoma and primary, patient-derived cells by flow cytometry. Additionally, it was shown to specifically distinguish carcinoma cells from other cell types present in whole blood samples, with the same degree of specificity as the EpCAM antibody. Hence, we propose that the developed aptamer might serve as a useful tool for detection of epithelial cancers. 


\section{Materials and Methods}

\section{Reagents and cell lines}

All cell lines used in this study were purchased from ATCC (Manassas, VA), unless specified otherwise. Cell lines propagated in suspension cultures were grown in RPMI1640 medium (Fisher Scientific, Pittsburgh, PA) supplemented with 10\% FBS (Atlanta Biologicals, Lawrenceville, GA). Cell lines grown in adhesion cultures were maintained in DMEM (Atlanta Biologicals) supplemented with $10 \%$ FBS.

The EpCAM-negative (non-epithelial) cell lines were: B-cell lymphoma cell lines CA46, Jeko-1, and Maver-1; T-cell lymphoma cell line U937; and T-cell leukemia cell line HEL, and K562. Epithelial cell lines used in these studies were prostate cancer cells PC3, VCaP, 22RV1, and DU145; ovarian cancer cells OVCAR3, Skov3, and Hey; breast cancer cells MDA-MB-231, MDA-MB-468, BT-474, and SKBR3; colon cancer cells HT29; and pancreatic cancer cells PANC-1, PANC-28, and ASPC-1 (kindly provided by Dr. Paul Chiao, MD Anderson Cancer Center, Houston, Texas).

\section{Aptamer selection procedure}

The DNA library used for aptamer selection consisted of a central, continuous stretch of 35 randomized nucleotides flanked by PCR primer sequences (5'-ATCCAGAGTGACGCAGCA-35NTGGACACGGTGGCTTAGT-3'). Two primers were used in the initial PCR reaction: Cy3-labeled $5^{\prime}$ primer (5'-cy3ATCCAGAGTGACGCAGCA-3') and biotinylated 3' primer (5'biotin-ACTAAGCCACCGTGTCCA-3'). The DNA library and primers were purchased from Integrated DNA Technologies (Coralville, IA), and the newly synthesized DNA library was purified by reversed-phase ion pairing high-performance liquid chromatography (Integrated DNA Technologies)

The aptamer synthesis and initial characterization were performed as previously reported [38]. Briefly, high-EpCAM-specificity aptamers were selected through a 3 -step process by exposing the ssDNA library to EpCAM-positive cell lines, EpCAM-positive primary colon epithelial cells, and recombinant EpCAM protein (Figure 1a). Briefly, the selection is performed step by step as below. DNA initial library incubation with EpCAM positive cells or proteins for $30 \mathrm{~min}$. -Washing away unbound DNA by DPBS buffer (Fisher Scientific) -Eluting bound DNA from cells or proteins by nuclease free water (Fisher Scientific) -- Amplifying eluted DNA by PCR-- Purified single strand (ss) DNA from PCR product by streptavidin beads -- Enriched ssDNA will be used for next round selection.

First, ssDNA clones were incubated with $5 \times 10^{6}$ EpCAM-positive MDA-MB-468 breast cancer cells for $30 \mathrm{~min}$ at room temperature. Following a wash with DPBS buffer (Fisher scientific) supplemented with $0.1 \%$ BSA, $0.1 \mathrm{~g} / \mathrm{L}$ tRNA, and $5 \mathrm{mM} \mathrm{MgCl}_{2}$, bound DNA was eluted by heating the mixture to $95^{\circ} \mathrm{C}$ for $10 \mathrm{~min}$. The eluted sequences were PCR-amplified using the Cy5-labeled forward and biotin-labeled reverse primers and the following cycle settings: denaturation at $94^{\circ} \mathrm{C}$ for $30 \mathrm{~s}$, annealing at $58^{\circ} \mathrm{C}$ for $30 \mathrm{~s}$, and extension at $72^{\circ} \mathrm{C}$ for $30 \mathrm{~s}$. The number of PCR cycles was optimized as below for each round of selection and ranged from 10 to 30: PCR products were separated on $2 \%$ agarose gels (Bio-Rad, Hercules, CA), and the highest cycle that displayed absence of nonspecific bands was chosen as the cycle number for subsequent amplification steps. Following amplification, ssDNA sequences were separated from the biotinylated antisense ssDNA by alkaline denaturation using $200 \mathrm{mM} \mathrm{NaOH}$, and then affinity purified with streptavidin-coated sepharose beads (Fisher Scientific). The separated ssDNA solution was passed through a desalting Nap-5 column (Fisher Scientific) to remove $\mathrm{NaOH}$, and the purified ssDNA was used for the next round of selection. Combined, this cell line-based selection was repeated five times.

In the second step, the enriched ssDNA pool was incubated with primary colon epithelial cells. Following incubation, the cells were washed with DPBS buffer (Fisher scientific), stained with PE labeled anti-EpCAM antibody (BD Biosciences, San Jose, CA), and sorted by fluorescence-activated cell sorting (NIR Arill, BD Biosciences). EpCAM-positive cells were collected, the bound DNA eluted and used for the next round of selection. Following 5 rounds of primary cell selection, the DNA was further enriched in step 3 via the incubation with a recombinant EpCAM protein: magnetic beads coated with Glutathione (Thermo Scientific) were pre-blocked with $5 \%$ bovine serum albumin (BSA, Fisher Scientific), washed with DPBS buffer, incubated with recombinant GST-EpCAM protein (Sino Biological, Beijing, China) for $30 \mathrm{~min}$, and washed again with DPBS buffer. Then, the EpCAM-coated beads were incubated with the enriched DNA pool for $30 \mathrm{~min}$, centrifuged for $5 \mathrm{~min}$ at $800 \mathrm{rpm}$, and washed twice. The eluted DNA then underwent two additional rounds of the recombinant protein-based selection.

Finally, after 13 rounds of cell- and protein-based selection, the resultant ssDNA pool was evaluated for its EpCAM binding affinity and specificity by flow cytometry (LSRII, BD Biosciences). Then, the selected ssDNA pool was sequenced using second-generation sequencing (LC Sciences, Houston, TX).

\section{Isolation of primary epithelial cells from fresh human tissues}

Freshly collected tissue (Obtained from Serum and tissue bank, Houston Methodist Hospital) was washed with PBS buffer (Fisher scientific), minced, and treated with $2 \mathrm{mg} / \mathrm{ml}$ collagenase/dispase solution (Sigma-Aldrich, St. Louis, MO) for $30 \mathrm{~min}$ at $37^{\circ} \mathrm{C}$. The resultant solution of dissociated cells was then sequentially filtered through $70 \mu \mathrm{m}$ and $45 \mu \mathrm{m}$ filters, and then centrifuged at $1000 \mathrm{rpm}$ for $5 \mathrm{~min}$ at room temperature. Cell pellets were resuspended in PBS buffer and used for staining with the aptamer or antibodies.

\section{Cell binding assays}

Staining of cells with aptamers and the subsequent flow cytometry analysis were carried out as previously reported [20]. Briefly, $1 \times 105$ cells were incubated with Cy3-labeled aptamers (Integrated DNA Technologies) for $30 \mathrm{~min}$ at room temperature, washed with PBS, and analyzed by the LSRII flow cytometer (BD Biosciences). Aptamer binding affinity was calculated using the reference method described previously [20,39]. The fluorescent ligand equilibrium dissociation constant $(\mathrm{Kd})$ was obtained by fitting the specific intensity $(\mathrm{Y})$ versus the aptamer concentration (X) plot to the $\mathrm{Y}=\mathrm{BmaxX} /(\mathrm{Kd}+\mathrm{X})$ equation using SigmaPlot (Jandel, San Rafael, CA).

To further confirm specificity of the newly-synthesized DNA aptamers, MDA-MB-468 (EpCAM-positive) and U937 (EpCAMnegative) cell lines were mixed in 1:1 ratio, and the mixture incubated for $30 \mathrm{~min}$ at room temperature with $100 \mathrm{nM}$ aptamer or EpCAM antibody conjugated to phycoerythrin (PE, BD Bioscience). Subsequently, the cell mixture was washed with PBS and EpCAM staining evaluated by flow cytometry (LSRII, BD Bioscience). In the last set of experiments, $1 \times 105$ MDA-MB-468 cells were mixed with 5 
$\mu \mathrm{l}$ whole blood or white blood cells (WBC) isolated from $500 \mu \mathrm{l}$ normal peripheral blood (obtained from serum and tissue bank, Houston Methodist Hospital), and the mixtures stained for $30 \mathrm{~min}$ with $100 \mathrm{nM}$ Cy5-labeled aptamer (Integrated DNA Technologies) and PE-EpCAM antibody at room temperature. Following a brief wash with DPBS buffer, the EpCAM-positive population was detected by flow cytometry (LSRII, BD Bioscience).

\section{Statistical Analysis}

For all studies, data were obtained from at least 3 independent experiments. Quantitative data are represented as mean \pm SD.

\section{Results}

Development and characterization of ssDNA aptamer sequences specific for EpCAM-expressing cells

To ensure aptamer EpCAM specificity and the ability to bind living cells, a ssDNA library containing random 35 nucleotides was synthesized and a 3-step hybrid SELEX was performed as illustrated in Figure 1a. Briefly, the initial aptamer selection was performed by exposing the ssDNA library to 5 sequential cycles of cultured EpCAMexpressing MDA-MB-468 breast cancer cells. In the second step, the enriched aptamer pool was further augmented by exposing it to 5 rounds of primary EpCAM-expressing colon epithelial cells. Thirdly, the enriched sequence pool was purified through three cycles of magnetic beads coated with recombinant EpCAM protein. Finally, the resultant aptamer pool was sequenced by the second-generation sequencing and the ratios of aptamer sequences were determined. As shown in Figure $1 \mathrm{~b}$, among over 500,000 total reads, there was a single dominant aptamer sequence that accounted for $58.7 \%$ of read frequency. Importantly, a multitude of minor clones with sequences that displayed a high degree of structural homology to the major sequence were also identified and are labeled as class 1 (Figure 1b). In addition, a clone that attributed to a significant $5 \%$ read frequency, but had a highly divergent sequence structure was also identified (class 2, Figure 1b).

For further analysis, two-dimensional structures of the developed class 1 (\#1-F, 71 bases) and class 2 (\#2-F, 71 bases) aptamers were predicted (Figure 2a). Both structures consisted of a unique central core and two primer regions, and contained well-formed loop and stem structures. To evaluate their targeting sensitivity, different concentrations of \#1-F and \#2-Faptamers were incubated with cultured EpCAM-positive MDA-MB-468 cells (468) and EpCAMnegative U937 control cells. As shown in Figure 2b, both \#1-F and \#2$\mathrm{F}$ aptamers bound to MDA-MB-468 cells with high binding affinity $(\mathrm{Kd}=12 \mathrm{nM})$ and exhibited minimal reaction to U937 control cells even at high (100 nM) concentrations. Next, white blood cells (WBC) isolated from normal peripheral blood were stained with \#1-F and \#2$\mathrm{F}$ aptamers at $100 \mathrm{nM}$ and analyzed by flow cytometry. As shown in Figure 2C, \#1-F aptamer displayed little WBC binding, as opposed to \#2-F that strongly bound to WBC cells. These data indicated that the \#1-F aptamer possesses a higher EpCAM specificity than \#2-F, prompting us to utilize \#1-F in all further studies.

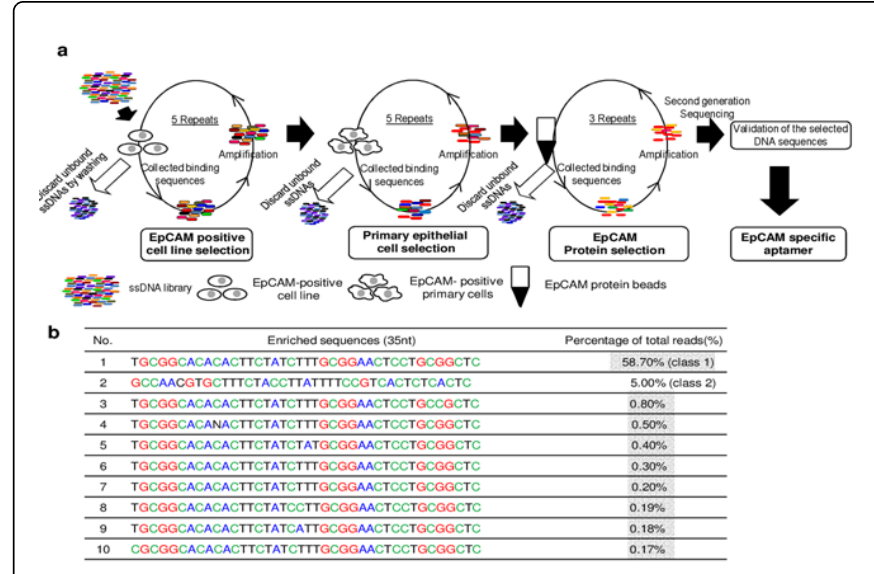

Figure 1: The aptamer selection process and sequencing results. (a) The aptamer selection process included five rounds of enrichment with EpCAM-positive cells, five rounds with primary epithelial cells, and three rounds with the recombinant EpCAM proteins. (b) Of 500,000 reads, the domain sequence accounted for $58.7 \%$ reads, while the second-most prevalent sequence accounted for $5 \%$.

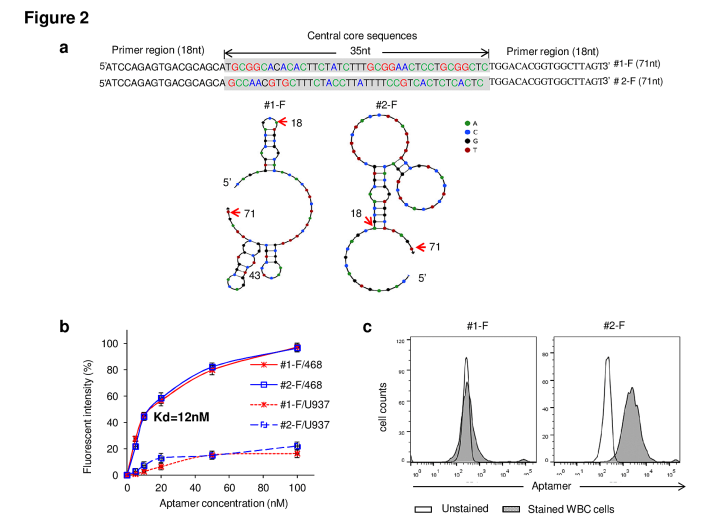

Figure 2: Characterization of the developed aptamers. (a)Predicted two-dimensional structures of Aptamer sequences of class 1 (\#1-F) and class $2(\# 2-\mathrm{F})$ aptamers, including 5 'primer, central core sequence, and 3' primer regions, and predicted two-dimensional structures. (b) EpCAM-positive (MDA-MB-468) and -negative (U937) cells were exposed to Cy5-labeled \#1-F and \#2-F aptamers at indicated concentrations and the resultant cell binding fluorescence was quantified by flow cytometry. (c) White blood cells isolated from normal peripheral blood were incubated with $\# 1-\mathrm{F}$ and \#2-F aptamers to rule out of target cell binding. All experiments were repeated by at least three times.

The EpCAM-specific aptamer preferentially targets cultured and patient-derived carcinoma cells. To further validate aptamer specificity, multiple cultured cancer cell lines were stained with the aptamer or an anti-EpCAM antibody as a control. As shown in Figure $3 \mathrm{a}$, the aptamer specifically recognized a variety of carcinoma cell 
Page 4 of 7

lines, including pancreatic (PANC-1), breast (MDA-MB-468 and SKBR-3), ovarian (Hey), prostate (DU145), and colon (HT-29), as well as two immortalized epithelial cell lines (293T and HeLa). Importantly, although EpCAM expression varied among different cancer types, the aptamer staining patterns were similar to those obtained with the anti-EpCAM antibody. Moreover, similar to the anti-EpCAM antibody, the aptamer did not react with EpCAMnegative cancer cells, such as T-cell lymphoma: U937, Acute myeloid leukemia: HEL and B-cell lymphomas (CA46 and Maver-1) (Figure $3 \mathrm{~b})$. A complete list of the aptamer cell binding studies performed in 23 cell lines is presented in Table 1.

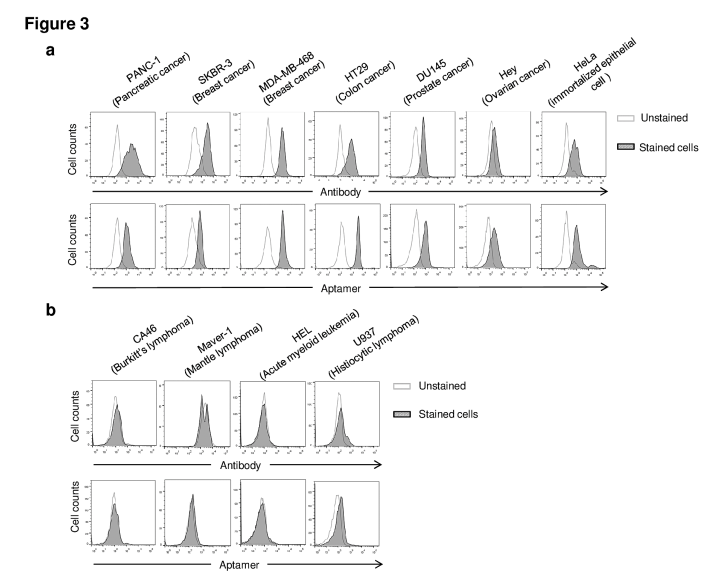

Figure 3: The\#1-F aptamer specifically recognizes EpCAMexpressing cancer cells. (a)Pancreatic (PANC-1), breast (MDAMB-468 and SKBR-3), ovarian (Hey), prostate (DU145), and colon (HT29) cancer cell lines, as well as two immortalized epithelial (293T and HeLa) cell lines were stained with \#1-Faptameror the anti-EpCAM antibody and the cell binding was determined by flow cytometry. (b) EpCAM-negative leukemia and lymphoma cell lines were examined by flow cytometry under the same condition.

\begin{tabular}{|l|l|l|l|}
\hline Cancer type & Cell line & $\begin{array}{l}\text { EpCAM } \\
\text { antibody }\end{array}$ & $\begin{array}{l}\text { EpCAM } \\
\text { aptamer }\end{array}$ \\
\hline \multirow{4}{*}{ Pancreatic cancer } & PANC-1 & ++ & ++ \\
\cline { 2 - 4 } & ASPC-1 & ++ & ++ \\
\cline { 2 - 4 } & PANC-28 & + & + \\
\hline \multirow{3}{*}{ Breast cancer } & MDA-MB-231 & ++ & ++ \\
\cline { 2 - 4 } & MDA-MB-468 & ++ & ++ \\
\cline { 2 - 4 } & BT-474 & + & + \\
\cline { 2 - 4 } & SKBR3 & ++ & ++ \\
\hline \multirow{3}{*}{ Ovarian cancer } & OVCAR3 & + & + \\
\cline { 2 - 4 } & SKOV3 & + & + \\
\cline { 2 - 4 } & Hey & + & + \\
\hline Prostate cancer & DU145 & ++ & ++ \\
\hline
\end{tabular}

Table 1: Summary of EpCAM aptamer binding to differentiate cancer cells.

To validate its potential for diagnostic clinical use, patient-derived normal epithelial and primary carcinoma cells isolated from fresh tissues (serum and tissue bank, Houston Methodist hospital) were then tested. It is important to note that the patient-derived cells were simultaneously co-stained with the anti-EpCAM aptamer and antibody in order to detect a dual-staining sub-population of cells. To do so, the cells were first gated based on the antibody staining, and then based on the aptamer binding (Figure 4). As shown in Figure 4c, this analysis identified sub-populations of cells that exhibited dual signal positivity, indicating that the newly synthesized aptamer possesses the sensitivity and specificity necessary for its clinical aptamer and antibody may target different epitopes of EpCAM molecules.

The EpCAM-specific aptamer selectively targets carcinoma, but not normal blood cells

Since the EpCAM-specific aptamer preferentially targeted patientderived primary carcinoma cells (Figure 4), it could be used in the future as a targeting component of a biotherapeutic. However, an aptamer used for in vivo targeted therapy must possess a high degree of specificity in addition to sensitivity. Specifically, an ideal aptamer will preferentially recognize only its target cells and display no crossreactivity to off-target normal cells or tissues. To address this n, a mixture of MDA-MB-468 breast cancer and U937 lymphoma cells was simultaneously treated with the EpCAM aptamer and the antiEpCAM antibody. Flow cytometric analysis revealed that both the aptamer and antibody highlighted the same breast cancer cell population, while exhibiting no reactivity to lymphoma cells (Figure 5a). Subsequently, 468 breast cancer cells were spiked into whole blood samples collected from healthy to mimic physiological conditions. The cancer cell-blood mixtures were then incubated with the anti-EpCAM aptamer and antibody and analyzed by flow cytometry, as described above. Alternatively, MDA-MB-468 breast cancer cells were also applications. In addition, cell staining results demonstrated that the 
Citation: Zhao N, Ge Y, Chiao J, Zu Y (2014) Development and Characterization of a ssDNA-based Aptamer that Selectively Targets Epithelial

Page 5 of 7

spiked into nucleated WBC cells and treated as above. Figures $5 \mathrm{~b}$ and $5 \mathrm{c}$ demonstrate that the EpCAM aptamer selectively targets breast cancer cells, but exhibits no reactivity with either the red blood or WBC cells, indicating its potential for the systemic in vivo use.

\section{Figure 4}

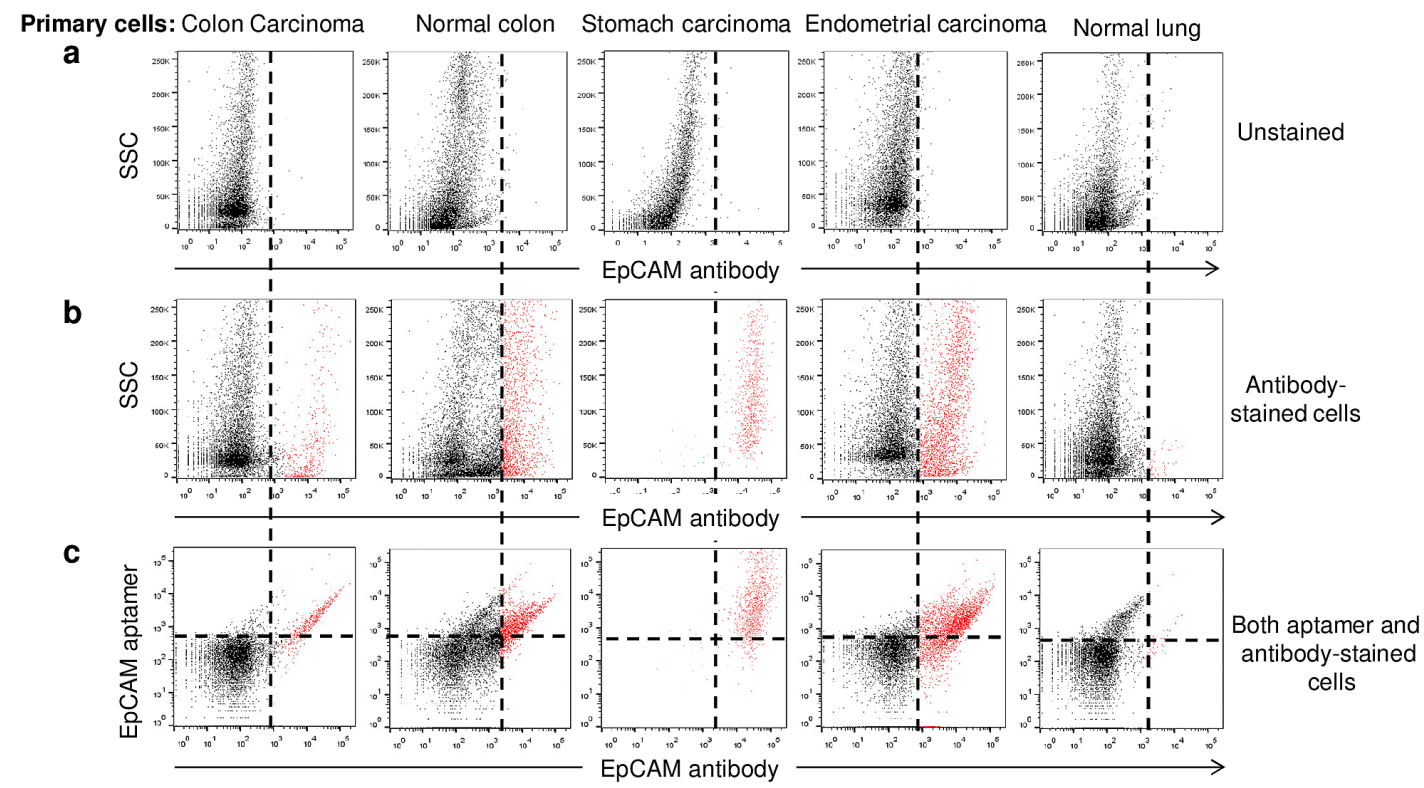

Figure 4: The \#1-F aptamer binds to primary epithelial and carcinoma cells. Patient-derived primary cells isolated from normal colon, colon carcinoma, normal lung, lung carcinoma, and stomach carcinoma tissues were dual-stained with the \#1-F aptamer and the anti-EpCAM antibody. Data shown are for unstained controls. (a) anti-EpCAM antibody-stained gate. (b) anti-EpCAM antibody and (c) aptamer dualstained cells.

\section{Discussion}

Aptamers, or "chemical antibodies", have recently emerged as a promising class of ligands that offer superb potential for diagnostic and therapeutic applications. Some of the aptamer advantages include ease of synthesis, small size, lack of immunogenicity, and versatile chemistry [40]. To date, Macugen or Pegaptanib, an aptamer targeting vascular endothelial growth factor (VEGF), has been the only aptamer approved by the FDA and is used to treat wet age-related macular degeneration. However, several other aptamer-based therapeutics are undergoing pre-clinical and clinical development [40]. In addition to its therapeutic potential, the aptamer technology could be utilized to develop novel imaging agents. For example, imaging of various diseases could be performed on the bases of targeting specific molecular markers, where aptamers offer unmatched capabilities. As such, these highly specific molecular aptamer probes could be used for detection of lesions, stratification of patients for clinical studies, evaluation of new drug toxicities and biodistribution, and treatment monitoring, among others [40-43].
In the present study, we describe a ssDNA-based aptamer that specifically targets EpCAM, a marker of epithelial and carcinoma cells. Our results show that the developed aptamer preferentially binds to EpCAM-positive, but not to EpCAM-negative cells at doses as high as $100 \mathrm{nM}$. Moreover, we demonstrate that the aptamer recognizes a variety of epithelial cancer cell lines, including pancreatic, prostate, ovarian, breast, and colon, as well as primary colon, Endometria, and stomach carcinoma cells, and normal primary colon and lung epithelial cells. Importantly, the EpCAM-specific aptamer clearly differentiated the EpCAM-positive cancer cells from other cell types present in the whole blood, which suggested that it could be used for detection of epithelial tumor cells in vivo, either to deliver targeted therapeutics or for epithelial tumor detection and imaging.

To elaborate on its potential for therapeutic or diagnostic use, the newly developed \#1-F aptamer can be used to detect epithelial carcinoma cells both, in vitro and in vivo. For in vitro applications, the aptamer can be conjugated with Fluorescein FAM or Cy3 dyes and subsequently used to detect EpCAM-positive cells by flow cytometry 
Citation: Zhao N, Ge Y, Chiao J, Zu Y (2014) Development and Characterization of a ssDNA-based Aptamer that Selectively Targets Epithelial

Page 6 of 7

or microscopy. For in vivo imaging, the aptamer can be labeled with long-wavelength Cy5 or IRD 800 dyes, which would enhance its tissue-penetrating abilities for sensitive detection of epithelial cancer cells. It is well known that, circulating tumor cells (CTCs), as a "liquid biopsy", can provide significant prognostic and predictive information for patient's clinical outcome and survival. However, current available detection of CTCs required an enrichment step to improve sensibility and/or specificity, Requirement of a multi-step cell preparation and isolation process may lead to loss and damage of tumor cells, and have an adverse impact on the assay accuracy, thereby limiting the clinical implementation of CTC tests and accurate interpretation of the test results. Due to our developed aptamer with non-reactivity with blood cells and strong sensitivity, it can be utilized for sensitive and specific detection of CTCs, without additional enrichment step. Finally, the highly specific \#1-F aptamer can be directly conjugated with a chemotherapeutic agent or combined with nanotechnology to establish novel, targeted approaches for treatment of carcinoma tumors. In summary, this EpCAM-specific aptamer technology offers a multitude of possibilities for research, diagnostic, and therapeutic applications.

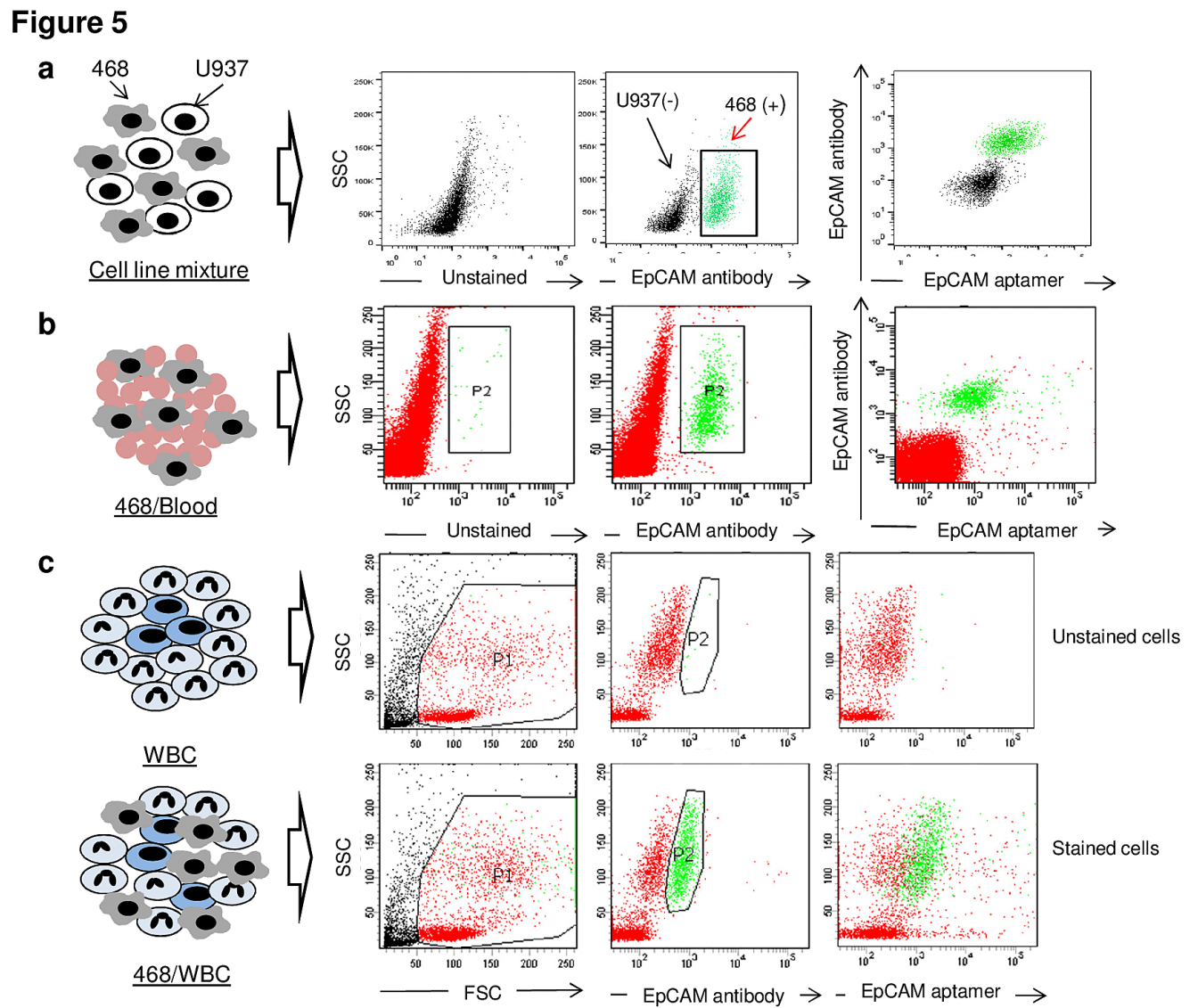

Figure 5: The EpCAM-specific \#1-F aptamer preferentially distinguishes epithelial cancer cells in cell mixtures. EpCAM-expressing MDAMB-468 cells were mixed with U937 control cells (a) or whole blood (b), stained with the \#1-F aptamer and the anti-EpCAM antibody. Resultant cell binding was examined by flow cytometry. (c) MDA-MB-468 cells were mixed with the nucleated white blood cells, and cell mixture was stained with the anti-EpCAM antibody and the \#1-F aptamer. Resultant cell binding was analyzed by flow cytometry. (Note: P1: gated living cells; P2: gated EpCAM-positive cells identified by EpCAM antibody)

\section{Acknowledgements}

This project was supported in part by grants R01CA151955 (Y.Z.) and R33CA173382 (Y.Z.) from the National Institute of Health. The authors are grateful to Yekaterina B. Khotskaya, Ph.D., for her help with editing of this manuscript.

\section{References}

1. Balzar M, Winter MJ, de Boer CJ, Litvinov SV (1999) The biology of the 17-1A antigen (Ep-CAM). J Mol Med (Berl) 77: 699-712.

2. Schnell U, Cirulli V, Giepmans BN (2013) EpCAM: structure and function in health and disease. Biochim Biophys Acta 1828: 1989-2001. 
3. Patriarca C, Macchi RM, Marschner AK, Mellstedt H (2012) Epithelial cell adhesion molecule expression (CD326) in cancer: a short review. Cancer Treat Rev 38: 68-75.

4. van der Gun BT, Melchers LJ, Ruiters MH, de Leij LF, McLaughlin PM, et al. (2010) EpCAM in carcinogenesis: the good, the bad or the ugly. Carcinogenesis 31: 1913-1921.

5. Stingl J, Raouf A, Emerman JT, Eaves CJ (2005) Epithelial progenitors in the normal human mammary gland. J Mammary Gland BiolNeoplasia 10: 49-59.

6. Baeuerle PA, Gires O (2007) EpCAM (CD326) finding its role in cancer. Br J Cancer 96: 417-423.

7. Trzpis M, McLaughlin PM, de Leij LM, Harmsen MC (2007) Epithelial cell adhesion molecule: more than a carcinoma marker and adhesion molecule. Am J Pathol 171: 386-395.

8. Ranganathan S, Schmitt LA, Sindhi R (2014) Tufting enteropathy revisited: the utility of MOC31 (EpCAM) immunohistochemistry in diagnosis. Am J Surg Pathol 38: 265-272.

9. Fartoux L, Decaens T (2011) Contribution of biomarkers and imaging in the management of hepatocellular carcinoma. Clin Res HepatolGastroenterol 35 Suppl 1: S21-30.

10. Hu M, Zhang K (2013) The application of aptamers in cancer research: an up-to-date review. Future Oncol 9: 369-376.

11. Liu J, You M, Pu Y, Liu H, Ye M, et al. (2011) Recent developments in protein and cell-targeted aptamer selection and applications. Curr Med Chem 18: 4117-4125.

12. Wang AZ, Farokhzad OC (2014) Current progress of aptamer-based molecular imaging. J Nucl Med 55: 353-356.

13. Wang T, Ray J (2012) Aptamer-based molecular imaging. Protein Cell 3: 739-754.

14. Thiviyanathan V, Gorenstein DG (2012) Aptamers and the next generation of diagnostic reagents. Proteomics ClinAppl 6: 563-573.

15. Ni X, Castanares M, Mukherjee A, Lupold SE (2011) Nucleic acid aptamers: clinical applications and promising new horizons. Curr Med Chem 18: 4206-4214.

16. Zhang Y, Chen Y, Han D, Ocsoy I, Tan W (2010) Aptamers selected by cell-SELEX for application in cancer studies. Bioanalysis 2: 907-918.

17. Stoltenburg R, Reinemann C, Strehlitz B (2007) SELEX--a (r)evolutionary method to generate high-affinity nucleic acid ligands. Biomol Eng 24: 381-403.

18. Ye M, Hu J, Peng M, Liu J, Liu J, et al. (2012) Generating Aptamers by Cell-SELEX for Applications in Molecular Medicine. Int J MolSci 13: 3341-3353.

19. Sefah K, Meng L, Lopez-Colon D, Jimenez E, Liu C, et al. (2010) DNA aptamers as molecular probes for colorectal cancer study. PLoS One 5: e14269.

20. Zhao N, Pei SN, Parekh P, Salazar E, Zu Y (2014) Blocking interaction of viral gp120 and CD4-expressing $\mathrm{T}$ cells by single-stranded DNA aptamers. Int J Biochem Cell Biol 51: 10-18.

21. Liang HR, Hu GQ, Li L, Gao YW2, Yang ST, et al. (2014) Aptamers targeting rabies virus-infected cells inhibit street rabies virus in vivo. Int Immunopharmacol 21: 432-438.

22. Tang Z, Parekh P, Turner P, Moyer RW, Tan W (2009) Generating aptamers for recognition of virus-infected cells. ClinChem 55: 813-822.

23. Savory N, Nzakizwanayo J, Abe K, Yoshida W, Ferri S, et al. (2014) Selection of DNA aptamers against uropathogenic Escherichia coli
NSM59 by quantitative PCR controlled Cell-SELEX. J Microbiol Methods 104: 94-100.

24. Gilch S, Schätzl HM (2009) Aptamers against prion proteins and prions. Cell Mol Life Sci 66: 2445-2455.

25. Zhang X, Zhang J, Ma Y, Pei X, Liu Q, et al. (2014) A Cell-based Singlestranded DNA Aptamer Specifically Targets Gastric Cancer. The international journal of biochemistry \& cell biology 46: 1-8.

26. Zhou W, Huang PJ, Ding J, Liu J (2014) Aptamer-based biosensors for biomedical diagnostics. Analyst 139: 2627-2640.

27. Santosh B, Yadava PK (2014) Nucleic acid aptamers: research tools in disease diagnostics and therapeutics. Biomed Res Int 2014: 540451.

28. Reinemann C, Strehlitz B (2014) Aptamer-modified nanoparticles and their use in cancer diagnostics and treatment. Swiss Med Wkly 144: w13908.

29. Zhao N, Bagaria HG, Wong MS, Zu Y (2011) A nanocomplex that is both tumor cell-selective and cancer gene-specific for anaplastic large cell lymphoma. J Nanobiotechnology 9: 2.

30. Kanwar JR, Shankaranarayanan JS2, Gurudevan S2, Kanwar RK2 (2014) Aptamer-based therapeutics of the past, present and future: from the perspective of eye-related diseases. Drug Discov Today 19: 1309-1321.

31. Xing H, Hwang K, Li J, Torabi SF, Lu Y (2014) DNA Aptamer Technology for Personalized Medicine. Curr Opin Chem Eng 4: 79-87.

32. Zhou J, Rossi JJ (2014) Cell-type-specific, Aptamer-functionalized Agents for Targeted Disease Therapy. Mol Ther Nucleic Acids 3: e169.

33. Hong B, Zu Y (2013) Detecting circulating tumor cells: current challenges and new trends. Theranostics 3: 377-394.

34. Li X, Zhao Q, Qiu L (2013) Smart ligand: aptamer-mediated targeted delivery of chemotherapeutic drugs and siRNA for cancer therapy. J Control Release 171: 152-162.

35. Zhang P, Zhao N, Zeng Z, Chang CC, Zu Y (2010) Combination of an aptamer probe to CD4 and antibodies for multicolored cell phenotyping. Am J ClinPathol 134: 586-593.

36. Zhang P, Zhao N, Zeng Z, Feng Y, Tung CH, et al. (2009) Using an RNA aptamer probe for flow cytometry detection of CD30-expressing lymphoma cells. Laboratory investigation; a journal of technical methods and pathology 89: 1423-1432.

37. Zeng Z, Zhang P, Zhao N, Sheehan AM, Tung CH, et al. (2010) Using oligonucleotide aptamer probes for immunostaining of formalin-fixed and paraffin-embedded tissues. Mod Pathol 23: 1553-1558.

38. Sefah K, Shangguan D, Xiong X, O'Donoghue MB, Tan W (2010) Development of DNA aptamers using Cell-SELEX. Nat Protoc 5: 1169-1185.

39. Meng L, Yang L, Zhao X, Zhang L, Zhu H, et al. (2012) Targeted delivery of chemotherapy agents using a liver cancer-specific aptamer. PLoS One 7: e33434.

40. Keefe AD, Pai S, Ellington A (2010) Aptamers as therapeutics. Nat Rev Drug Discov 9: 537-550.

41. Gross S, Piwnica-Worms D (2006) Molecular imaging strategies for drug discovery and development. Curr Opin Chem Biol 10: 334-342.

42. Hong H, Goel S, Zhang Y, Cai W (2011) Molecular imaging with nucleic acid aptamers. Curr Med Chem 18: 4195-4205.

43. Soontornworajit B, Wang Y (2011) Nucleic acid aptamers for clinical diagnosis: cell detection and molecular imaging. Anal Bioanal Chem 399: 1591-1599. 